\title{
Giant parametric amplification of the nonlinear response in a single crystal of beryllium in a quantizing magnetic field
}

\author{
M.I. Tsindlekht ${ }^{1,2}$, N. Logoboy ${ }^{1,2}$, V.S. Egorov ${ }^{1,3}$, R.B.G. Kramer ${ }^{1}$, \\ A.G.M. Jansen ${ }^{4}$, and W. Joss ${ }^{1}$ \\ ${ }^{1}$ Grenoble High Magnetic Field Laboratory, Max-Plank-Institute für Festkörperforschung, \\ B.P. 166X, Cedex 9, Grenoble F-38042, France \\ ${ }^{2}$ The Racah Institute of Physics, The Hebrew University of Jerusalem, Jerusalem 91904, Israel \\ Email: mtsindl@vms.huji.ac.il \\ ${ }^{3}$ Russian Research Center Kurchatov Institute, Moscow, 123182, Russia \\ ${ }^{4}$ Service de Physique Statistique, Magnetisme et Supraconductivity, DRFMC, \\ CEA, 17 rue de Martyrs, Cedex 9, Grenoble, F-38054, France
}

Received March 3, 2006

\begin{abstract}
The nonlinear response to an ac magnetic field with and without parametric pumping was studied experimentally on a rod-like beryllium single crystal at low temperatures in a quantizing magnetic field applied parallel to the hexagonal axis. At low temperatures $(\leq 3 \mathrm{~K})$ giant parametric amplification of the nonlinear response is observed in narrow windows near de Haas- van Alphen (dHvA) beating antinodes where the amplitude of the magnetic oscillations is maximal. This effect is of threshold character with respect to the amplitude of the dHvA oscillations and occurs as a result of a diamagnetic phase transition of a strongly correlated electron gas into nonuniform state with formation of Condon domain structure. It is shown that the nonuniform phase appears periodically in a magnetic field. The components of rectified signal field reproduce the envelope of $H-T$ critical curves and can be used for reconstruction of diamagnetic phase diagrams.
\end{abstract}

PACS: 71.70.Di, 74.25.Nf, 74.60.Ec, 75.60.-d

Keywords: de Haas - van Alphen effect, Condon domains, paramrtric phenomena.

\section{Introduction}

Quantum oscillations of resistivity and magnetization in bismuth were discovered in 1930 by Schubnikov and de Haas [1] and de Haas and van Alphen, respectively [2]. The observation of quantum oscillations in other than bismuth metals was reported about ten years later after the first publications. Very important works concerning the the Schubnikov-de Haas and de Haas - van Alphen effects in single crystals of zinc, tin, beryllium were published by the group of B.G. Lazarev [3]. Only in the early fifties was it understood that quantum oscillations are a powerful tool for the study of the electronic structure of metals. Since that time the experiment and theory of quantum oscillations in metals at low temperatures were improved and detailed information about the Fermi surfaces of metals was obtained. However, even now there are a lot of loose ends and unsolved problems in this part of low-temperature physics. Nonlinear phenomena in a quantizing magnetic field are one of these problems.

Nonlinear properties of normal metals with a long mean free path of electrons were studied in detail about 35 years ago. It was understood that for the observation of a noticeable nonlinear response in a quantizing magnetic field $H$, the ac field amplitude $h_{0}$ has to be comparable to the period of the magnetization oscillations $\Delta H=H^{2} / F$, where $F$ is the de Haas-van Alphen (dHvA) frequency [4]. This means that at low amplitudes of excitation, when $h_{0}<\Delta H$, the amplitude of the nonlinear response should be small, and this phenomenon could be well described in the frame of perturbation theory [5-7]. Perturbation theory provides a good explanation for the majority of nonlinear experi- 
ments with normal metals in low magnetic fields; see for example [8] and references therein. The nonlinear helicon resonance in a quantizing magnetic field was studied in aluminium [9]. Experiments show that resonance curve shifts when the amplitude of the excitation in the metal exceeds the dHvA period. A complete theory of nonlinear helicon resonance does not exist up to now. The recent experimental study of the dHvA oscillations in single crystal beryllium at low temperatures showed that strong nonlinear response appears even at very low amplitude of excitation in an antinode area of dHvA oscillations [10].

All the above mentioned experiments are related to nonlinear effects, such as self-influence [9], harmonic generation, and/or rectification $[8,10]$. Another class of nonlinear effects is related to parametric phenomena. Parametric phenomena are important in fundamental physics and for applications. For example, Josephson junctions have been used as parametric amplifiers of weak microwave signals [11]. Experimental observations of parametric amplification of the weak microwave signals were possible because of the nonlinearity of the inductance of the Josephson junction [11]. Recently, giant parametric amplification of the nonlinear response was observed in the surface superconducting state, for $H_{c 2}<H<H_{c 3}$, of niobium single crystals [12,13]. Application of parametric pumping in the latter case leads to a two order-of-magnitudes enhancement of the nonlinear response which cannot be described by perturbation theory. The main physical reason for the observed phenomenon is a transition between metastable states in a surface superconducting state upon application of an ac magnetic field $[11,13]$.

In this paper we present the results of an experimental study of parametric phenomena in a nonsuperconducting single crystal of beryllium in a quantizing magnetic field. It is well known, that the amplitude of the dHvA effect in beryllium in a quantizing magnetic field is very high [4]. The conditions of diamagnetic phase transition, where instabilities appear in the system, develop periodically with variation of the magnetic field. As a result, the line shape of the dHvA oscillations, $M(H)$, becomes sawtooth-like in rod-like samples (Shoenberg effect), while in plate-like samples, Condon domains form $[4,15]$. Such a sawtooth shape of magnetization is somewhat similar to flux jumps in superconductors mentioned above. However, parametric phenomena in normal metals in a quantizing magnetic field, to the best of our knowledge, have not been studied either experimentally or theoretically so far. Therefore, it is interesting to study parametric phenomena in normal metals too. The best candidate for this role is beryl- lium which has strong dHvA effect. The relatively high amplitude of dHvA oscillations in beryllium is explained by the cylinder-like Fermi surface. It has well known folders of electrons «cigar» with two extremal cross-sections of almost equal areas. Consequently, the dHvA oscillations show a beat with a period 33 times larger than the fundamental frequency period. Due to this effect, at constant temperature the amplitude oscillations of magnetization can be changed drastically by small changes in dc magnetic field.

\section{Experimental setup}

We have intended to apply the technique used in the experiment with $\mathrm{Nb}[12,13]$ for the study of parametric phenomena in beryllium. A rectangular $(8 \times 1 \times 1 \mathrm{~mm})$ sample was cut by an electric spark from a high quality single crystal beryllium bar (the same bar which was used for the sample on which diamagnetic domains were observed in [16]). The long axis of the sample is parallel to the [0001] direction. Measurements of the rectified signal were carried out as follows (see $[12,13]$ for details). The sample was excited by an amplitude modulated high frequency field $h(t)=h_{0}(1+\alpha \cos \Omega t) \cos \omega t$. A small high frequency coil was used for this purpose. Typical values of the above parameters were $h_{0}<1.2$ Oe, $\alpha \approx 0.9$, $\omega / 2 \pi=1.4 \mathrm{MHz}$, and $\Omega / 2 \pi=1609 \mathrm{~Hz}$. When the ac excitation is applied to a sample in the nonlinear state, the magnetic moment of the sample oscillates at the harmonics of the fundamental frequency $\omega$, at frequencies $\omega \pm \Omega$, and at the frequency of modulation $\Omega$ and its harmonics. Secondary coils convert these

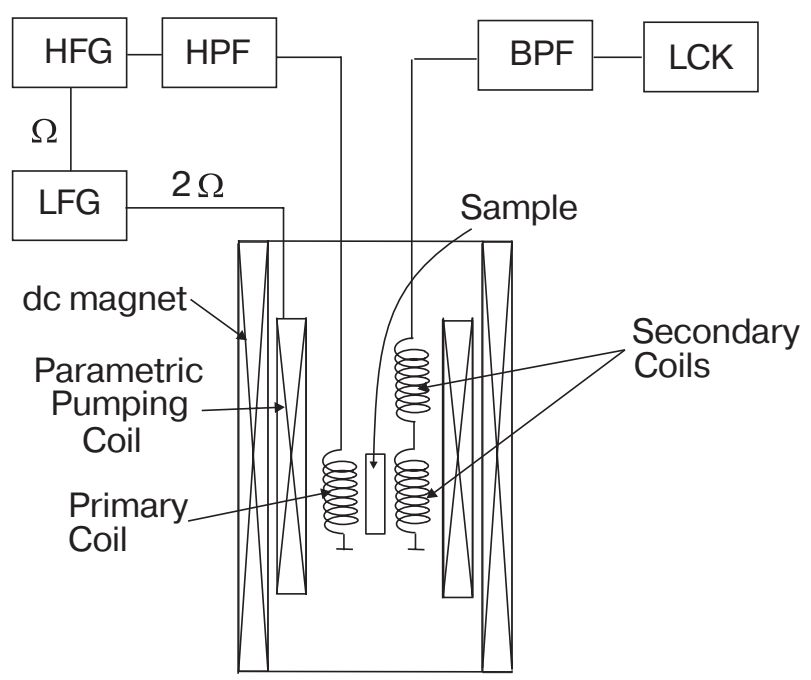

Fig. 1. Block diagram of the experimental setup. HFC is high frequency generator, LFG is low frequency generator, HPF and BPF is high and band pass filters, LCK is lock-in amplifier. 
magnetic moment oscillations into an ac voltage signal. In addition, a parametric pump field $h_{p}(t)=$ $=h_{2 \Omega} \cos \left(2 \Omega t+\Psi_{2 \Omega}\right)$ was applied using a separate copper driving coil.

We would like to emphasize the difference between our and standard parametric experiments. Usual parametric experiments have to deal with two signals. The system is excited by weak and strong (pump) signals. The frequency of the pump signal is twice as large as the frequency of the weak signal. Due to the nonlinearity of the system, power from the pump signal could be transferred to the weak signal [17]. In our experiment the sample of beryllium with nonlinear $M(B)$ dependence was excited by an amplitude modulated ac field with carrier frequency $\omega$ and modulation frequency $\Omega$. The spectrum of this ac field does not contain frequency $\Omega$. A signal with frequency $\Omega$ in a system appears when the sample is in a nonlinear state. Application of parametric pumping with frequency $2 \Omega$ could lead to amplification of the rectified signal on the frequency $\Omega$.

In the nonlinear experiments the amplitude $A_{\Omega}$ and the phase $\Psi_{\Omega}$ of the signal at frequency $\Omega$ as a function of the dc magnetic field $H$, ac field amplitude $h_{0}$, amplitude $h_{2 \Omega}$ and phase $\Psi_{2 \Omega}$ of the parametric pump $h_{2 \Omega}$ have measured by means of lock-in detection. A block diagram of the experimental setup is shown in Fig. 1. A primary high frequency copper coil, driven by a high frequency generator, produces an amplitude modulated ac field $h(t)$. Signals with frequencies $\Omega$ and $2 \Omega$ were provided by another generator. The modulation frequency $\Omega$ was used as a reference frequency for the lock-in amplifier. The ac field amplitudes $h_{0}$ and $h_{2 \Omega}$ were measured by an additional small probe coil wound under the primary coil. For the sake of clarity the probe coil is not shown in Fig. 1. The high-pass and band-pass filters are a crucial part of this set-up (Fig. 1). These filters should be passive and they have to prevent (i) the leak of the hindrance signal of frequency $\Omega$ through the high frequency generator (high-pass filter) and (ii) penetration of signals of frequency $\omega$ and $2 \Omega$ to the input of the lock-in amplifier (band-pass filter). One should note that the ratio between the pumping frequency and the modulation frequency was exactly equal to 2 in our experimental setup.

\section{Experimental results}

The linear susceptibility $\chi=d M / d H$ was measured by the pick-up coils method [4] at $22 \mathrm{~Hz}$ using the same coil system. The field dependence of the $\chi$ at $4.2 \mathrm{~K}$ is shown in Fig. 2,a. The observed spectrum is typical for the dHvA effect in beryllium for the $\mathbf{H} \|$ [0001] direction. Analysis shows that this spectrum consists of two
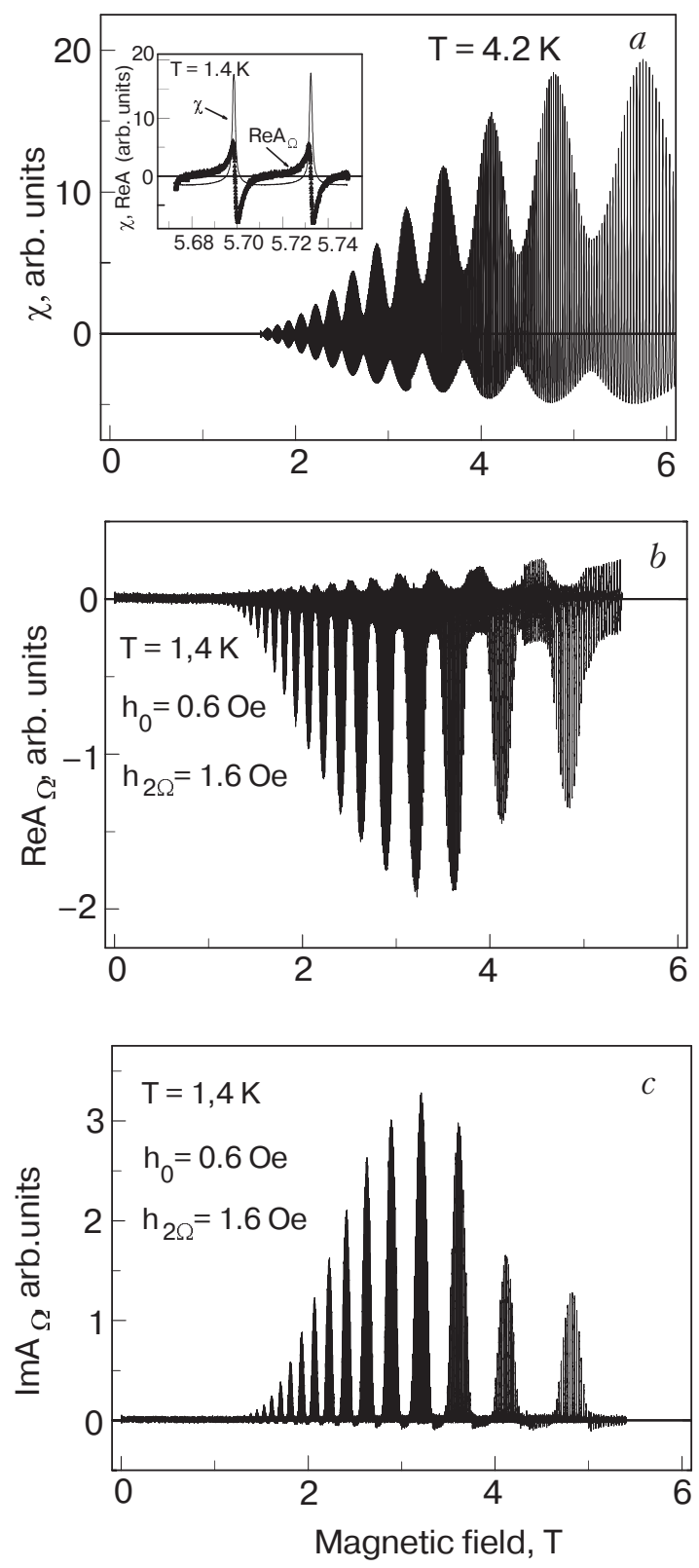

Fig. 2. Linear susceptibility $\chi$ of beryllium single crystal with [0001] || $\mathbf{H}$ versus magnetic field. Inset: Field dependence of $\chi=d M / d H$ and in-phase signal, $\operatorname{Re} A_{\Omega}$, without parametric pump (a). In-phase, $\operatorname{Re} A_{\Omega}$, component of rectified signal field dependence $(b)$. Out-of-phase, $\operatorname{Im} A_{\Omega}$, component of rectified signal field dependence $(c)$.

dHvA frequencies, $\quad F_{1} \simeq 940 \mathrm{~T}$ and $F_{2} \simeq 970 \mathrm{~T}$, corresponding to two extremal cross-sections of the «cigar»-like Fermi surface $[4,15]$. Decreasing the temperature leads to a sharp increase of the amplitude of the dHvA oscillations (see inset of Fig. 2,a). In the present work we measure the nonlinear properties of beryllium at $T \leq 1.4 \mathrm{~K}$.

Measurements of the in-phase component of the rectified signal, $\operatorname{Re} A_{\Omega}$, (inset to Fig. 2,a) show that its shape looks like the first derivative of the susceptibility $\chi$ and 


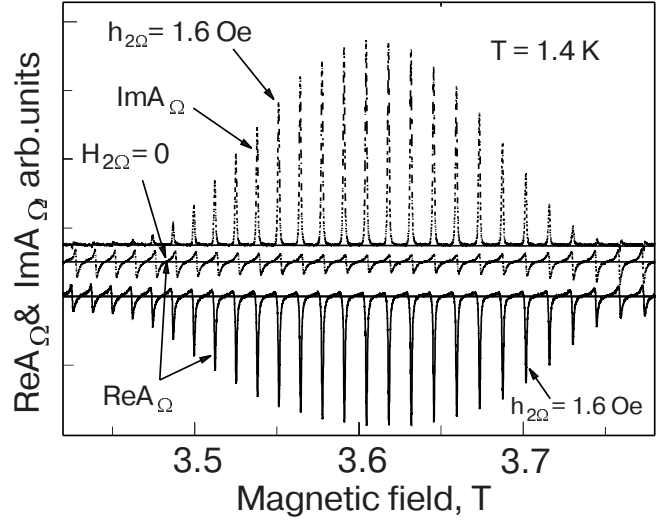

Fig. 3. Re $A_{\Omega}$ and $\operatorname{Im} A_{\Omega}$ signals versus magnetic field near antinode of magnetization beat.

the amplitude dependence of $A_{\Omega}\left(h_{0}\right)$ is almost quadratic. The amplitude of the out-of-phase component of signal, Im $A_{\Omega}$, is much smaller than $\operatorname{Re} A_{\Omega}$. This means that perturbation theory can be used for an explanation of the experimental observations presented above, and the ratio $h_{0} / \Delta H \ll 1$ is a «good» small parameter for this problem.

Application of parametric pumping introduces an essential change into the nonlinear response. Figures 2,b, and 2,c show field dependencies of the Re $A_{\Omega}$ and $\operatorname{Im} A_{\Omega}$ components of the rectified signal in the presence of parametric pumping.

Parametric pumping affects the rectified signal only at low temperatures and for magnetic fields near the antinodes of the magnetization beat and does not affect nonlinear response near the nodes of the beats. In magnetic fields $H>6 \mathrm{~T}$ the application of parametric excitation does not produce any changes in rectified signal. For $T=4.2 \mathrm{~K}$ parametric pumping has no influence on the nonlinear response at any values of magnetic field.

Figure 3 presents the field dependence of $\operatorname{Re} A_{\Omega}$ and $\operatorname{Im} A_{\Omega}$ with and without parametric pumping near an antinode of the beat in more detail. First of all, as mentioned above, $\operatorname{Re} A_{\Omega}$ without parametric pumping is small and looks like the first derivative of $\chi$. With the application of parametric pumping the rectified signal becomes unusual for magnetic fields near the antinode, $3.49 \mathrm{~T}<H<3.74 \mathrm{~T}$. In this region, at a given $h_{0}$ and $h_{2 \Omega}$, the signal grows steeply with the dc magnetic field, whereas the dHvA amplitude changes smoothly. This behavior leads us to speculate about the threshold character of this phenomenon with respect to the dHvA amplitude.

Figure 4 shows the field dependence of $\operatorname{Re} A_{\Omega}$ and $\operatorname{Im} A_{\Omega}$ in the small region near the maximum of the dHvA at different pump amplitudes, $h_{2 \Omega}$. Parametric pumping affects the out-of-phase component of the rectified signal more strongly than the in-phase one.
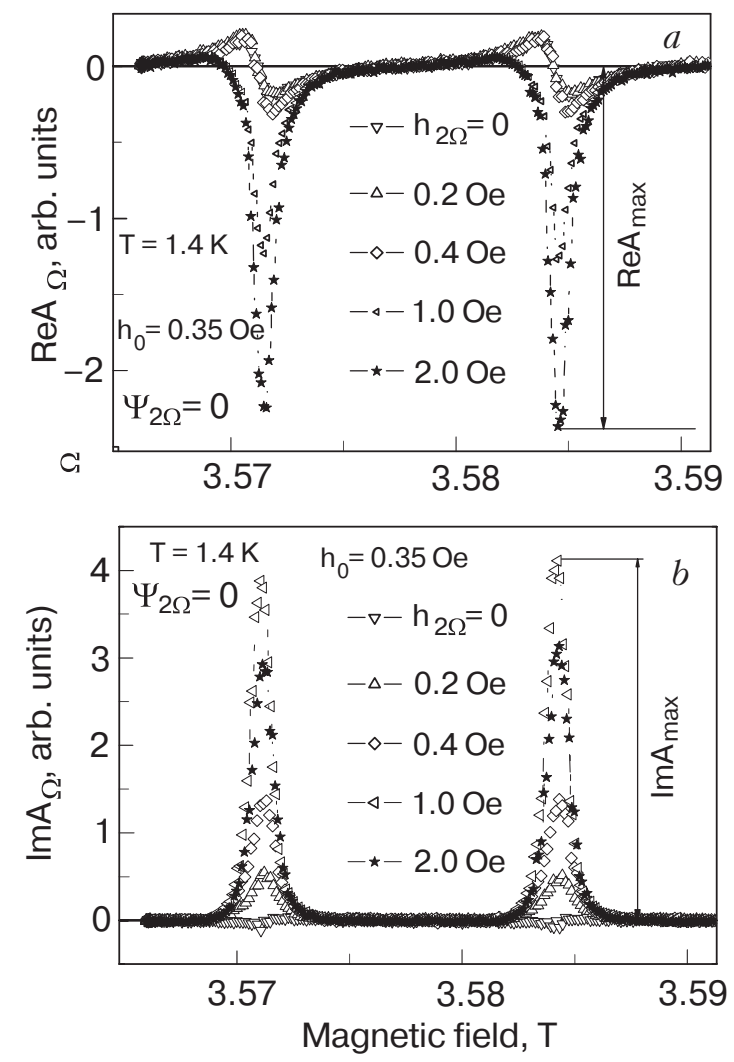

Fig. 4. Re $A_{\Omega}$ versus magnetic field $(a)$. Im $A_{\Omega}(H)$ for different amplitude $h_{2 \Omega}$ and $\Psi_{2 \Omega}=0(b)$.

In Fig. 5 we present the magnitude of $\operatorname{Re} A_{\Omega}$ and $\operatorname{Im} A_{\Omega}$ as a function of $h_{2 \Omega}$ for a magnetic field near the beating maximum $H=3.6 \mathrm{~T}$ and for $H=5.8 \mathrm{~T}$. Both $\operatorname{Re} A_{\Omega}$ and Im $A_{\Omega}$ increase almost quadratically at low pump levels $h_{2 \Omega} \leq 1$ Oe. Perturbation theory predicts a linear dependence at low amplitude of the parametric pump [17]. With further increase of the pump $h_{2 \Omega}$ the amplitudes don't change significantly, showing only a small decrease. Therefore, there seems to be an optimal parametric pump level of about 1 Oe.

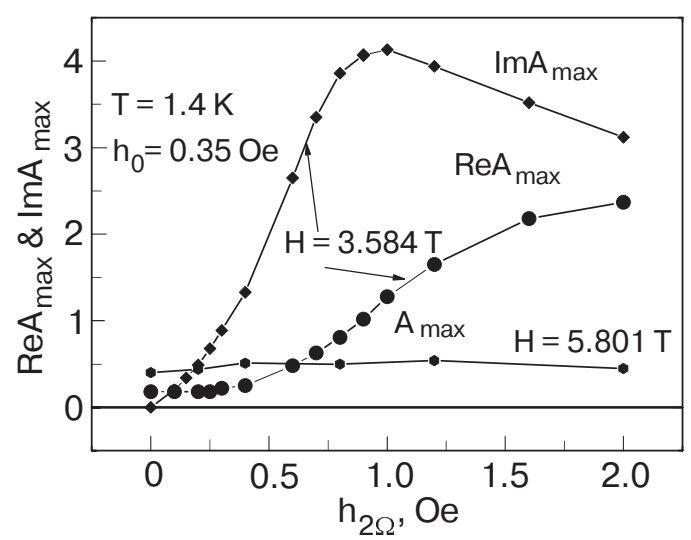

Fig. 5. Re $A_{\max }$ and $\operatorname{Im} A_{\max }$ versus $h_{2 \Omega}$ near the beat maximum $(H=3.584 \mathrm{~T})$ and $A_{\max }$ for $H=5.801 \mathrm{~T}$. 


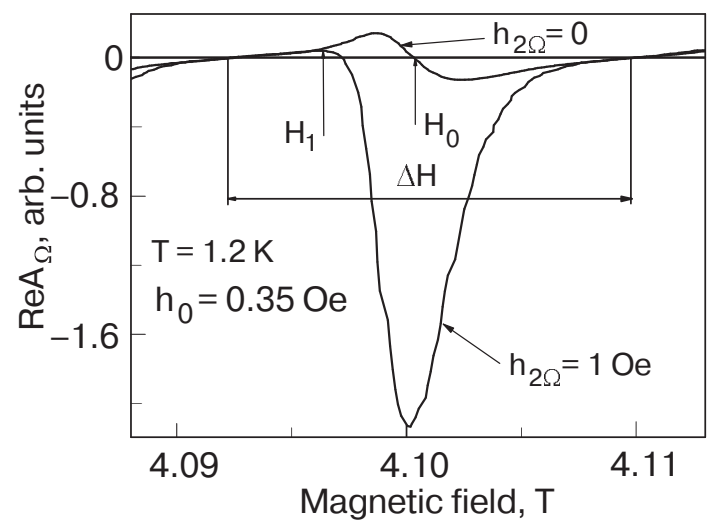

Fig. 6. Field dependence of $\operatorname{Re} A_{\Omega}$ with and without parametric pumping for one dHvA oscillations period.

At $H=5.8 \mathrm{~T}$ parametric pumping almost does not affect the rectified signal.

\section{Discussion}

Diamagnetic phase transition into a nonuniform (Condon domains) phase is responsible for the observed giant paramagnetic amplification of the nonlinear response. The currently available experimental data allow us to roughly define the range of magnetic fields in the dHvA period where a nonuniform phase exists. Figure 6 presents the field dependence of the rectified signal without and with parametric pumping. The line of the rectified signal is nonsymmetric. One can readily see that parametric pumping begins to affect strongly the rectified signal for magnetic field when $H>H_{1}$. Using these experimental data one can measure the value of the dHvA period itself, $\Delta H$, and the center of the dHvA period, $H_{0}$. The relative part of the dHvA period occupied by nonuniform phase, $\delta H / \Delta H$ can be roughly determined for each dHvA period. Here $\delta H=2\left(H_{0}-H_{1}\right)$. Figure $7, a$ shows $\delta H / \Delta H$ as a function of $H_{0}$. The edge experimental points, $\delta H / \Delta H=0$, were determined from the dHvA oscillation when the changes of the rectified signal line due to parametric pumping are negligible small.

The technique of parametric pumping amplification of the nonlinear response in single crystal of beryllium allows us to define the critical values of the magnetic field, when the amplitude of the dHvA oscillations becomes high enough for realization of a diamagnetic phase transition. It is interesting to compare the experimental curve of Fig. 7, $a$ with theoretical calculations.

The theoretical description of experimental data should be based on construction of diamagnetic $H-T$ phase diagrams. Undoubtedly, for correct explanation of the relatively high amplitude of the dHvA oscillations, the real shape of the Fermi surface of beryllium has to be taken into account. The Fermi surface of beryllium was investigated very carefully in the past [4]. This Fermi surface differs significantly from the free electron model and consists only of the first and second zone monster («coronet») and the third zone «cigar». Three maximum cross-sections of the «cigar» («waist» and «hips») are characterized by a small curvature, which results in relatively high amplitude of dHvA oscillations. Thus, the standard approach, based on Lifshitz-Kosevich formalism [4] giving satisfactory results for a free electron gas, fails to describe the electron instability in beryllium. Clearly, the anomalously low curvature of the Fermi surface of beryllium near the extremal cross-sections has to be taken into account. The model developed in [20] is a more realistic one. It can be considered as an attempt to take into account the actual 3D Fermi surface geometry for beryllium. It is consistent with the previous experiments on observation of the Condon instability at a value of the magnetic field up to $\sim 3 T$. However, it gives overestimated values for higher field and inconsistent with our present experiments and recent publi-
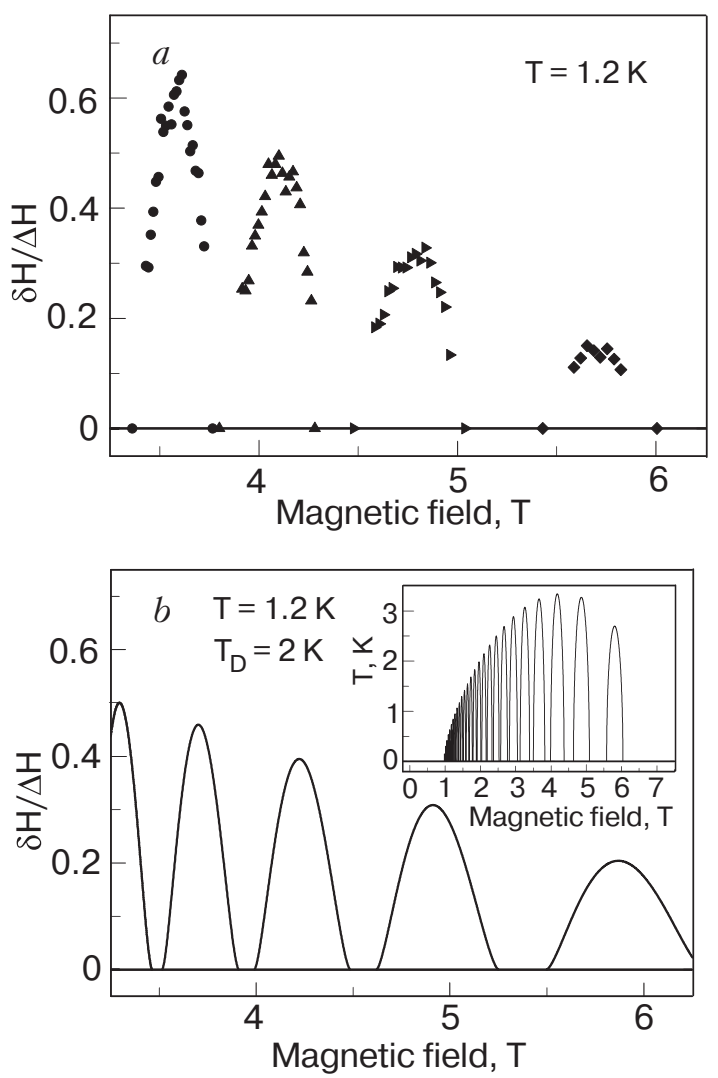

Fig. 7. The field dependence of $\delta H / \Delta H(a)$. This ratio defines the part of dHvA period occupied by nonuniform phase. The calculated ratio of the range of existence of the Condon domains $\delta H$ to the period of dHvA oscillations $\Delta H(b)$. The typical envelope of the $H-T$ phase diagram of beryllium for Dingle temperature $T_{D}=2 \mathrm{~K}$ is shown on the inset. 
cation on the observation of Condon domains in beryllium [10]. Our analysis of experimental data is based on the recent theory of diamagnetic phase transitions [22], which allows one to reconstruct the phase diagrams consistent with present experimental results.

For every period of dHvA oscillations when the reduced amplitude of oscillations $a \equiv d M / d B \geq 1$, the instability of an electron gas, e.g. the diamagnetic phase transition, results in the formation of a magnetization jump for an infinitely long cylinder or of Condon domain structure in the plate-like sample with strong temperature and magnetic field dependencies of the local magnetic moments [21]. In the domain phase the metastable states respect to the magnetic field are determined by $\hat{\partial} \Phi / \partial M=0$ with $\hat{\partial}^{2} \Phi / \partial M^{2}>0$, identifying the local minima of the thermodynamic potential $\Phi$ relatively to the magnetization $M$. In the center of the period of dHvA oscillations $\left(H=H_{0}\right)$ the two minima have the same energy corresponding to the remnant magnetization. The instability points are points where the two conditions $\partial \Phi / \partial M=0$ and $\partial^{2} \Phi / \partial M^{2}=0$ must simultaneously hold, e.g. inflection points, restrict the range of existence of the nonuniform phase. According to [21] the ratio of this range $\delta H$ to the period of dHvA oscillations $\Delta H$ is defined as follows

$$
\delta H / \Delta H=\frac{1}{\pi}\left(\sqrt{a^{2}-1}-\arccos \frac{1}{a}\right) .
$$

The results of calculation of $\delta H / \Delta H$ Eq. (1) in the model of a slightly corrugated cylinder Fermi surface of beryllium [22] are represented in Fig. 7,b. In the inset of Fig. 7,b the typical theoretical phase diagram for beryllium is shown. It can be emphasized that the in-phase and out-of phase components of rectified signals (Fig. 2), being dependent on the phase state of strongly correlated 3D electron gas, recall the shape of the phase diagrams, reproducing the envelope of the diamagnetic phase curves $H-T$ (see, inset of Fig. 7,b). There is a rather good agreement between experimental data (Fig. 7,a) and the theoretical calculations (Fig. $7, b$ ). Thus, the technique of parametric pumping is a powerful tool for reconstructing the phase diagrams of the diamagnetic metals under the conditions of a strong dHvA effect.

\section{Conclusions}

Though the dHvA effect itself is a strong nonlinear phenomenon, perturbation theory can be applied for an explanation of the rectification without parametric pumping even at low temperatures. In this sense beryllium is a weak nonlinear system. However, at low temperatures application of parametric pumping leads to giant amplification of the nonlinear response in nar- row windows of the dc magnetic field near the beating antinodes in a part of dHvA period (see Fig. 4) when the diamagnetic phase transition takes place $[18,19]$. The period of the dHvA oscillations grows as $H^{2}$ and the diamagnetic phase transition becomes less steep. At the values of magnetic fields, $H \geq 6 \mathrm{~T}$, parametric pumping does not affect the rectified signal, which is consistent with recent theoretical considerations [22]. The non-linear problems of harmonic generation beyond perturbation theory, under conditions of a strong dHvA effect were discussed theoretically long ago [7]. However, direct application of this theory to observed parametric phenomena is not possible at the present time. For a detail comparison of the experimental findings presented in this paper and the detailed theory of the non-linear response with parametric pumping has to be developed.

The giant parametric amplification of nonlinear response was detected in the surface superconducting state of niobium and in normal metal beryllium under conditions of a strong dHvA effect. Both systems demonstrate: (i) a phase transition between metastable states in niobium and (ii) diamagnetic phase transition in beryllium. One can believe that we have to deal with a general phenomenon inherent to systems with phase transitions. This phenomenon could be used for the experimental reconstruction of the $H-T$ phase diagram of a system with a phase transition.

It is supposed, also, that the parametric amplification of the nonlinear response may be observed in any nonlinear system. In our experiments with niobium in a sweeping magnetic field [23] and with beryllium in the present study we show that nonlinearity of the system itself is a necessary but not a sufficient condition for the observation of the parametric phenomena.

We thank Professor I. Vagner and Dr. I. Sheikin for useful discussions. One of the authors (M.I.T.) is grateful for the support of the European Community from the contract RITA-CT-2003-505474.

1. L. Schubnikov und W.J. de Haas, Leiden Commun. 207D (1930), ibid. 210A (1930).

2. W.J. de Haas und P.M. van Alphen, Leiden Commun. 212A (1930) ibid. 220D (1932).

3. B.G. Lazarev, N.M. Nakhimovich, and E.A. Parfenova, ZhETP 9, 1169 (1939); ibid. 9, 1182 (1939); B.I. Verkin, B.G. Lazarev, and N.S. Rudenko, Dokl. Akad. Nauk SSSR 69, 773 (1949).

4. D. Shoenberg, Magnetic Oscillations in Metals, Cambridge University Press, Cambridge (1984).

5. M.Ya. Azbel' and G.A. Begiashvili, Pis'ma ZETP, 3, 201 (1966), [JETP Lett. 3, 128 (1966)].

6. R.G. Mints, ZhTF Pis. Red. 11128 (1970).

7. L.B. Dubovsky, Zh. Eksp. Teor. Fiz. Sov. 58, 2111 (1970).

8. V.T. Dolgopolov, Sov. Phys. Usp. 23, 134 (1980). 
9. V.T. Petrashov, Rep. Prog. Phys. 47, 47 (1984).

10. R.B.G. Kramer, V.S. Egorov, A.G.M. Jansen, and W. Joss, Phys. Rev. Lett. 95, 187204 (2005).

11. A. Barone and G. Paterno, Physics and Applications of the Josephson Effect J. Wiley \& Sons, New York (1982).

12. M.I. Tsindlekht and I. Felner, Physica B329, 1371 (2003).

13. M.I. Tsindlekht, I. Shapiro, and M. Gitterman, Supercond. Sci. Technol. 18, 824 (2005).

14. R.W. Rollins and J. Silcox, Phys. Rev. 155, 404 (1967).

15. J.H. Condon, Phys. Rev. 145, 526 (1966).

16. G. Solt, C. Baines, V.S. Egorov, D. Herlach, and U. Zimmermann, Phys. Rev. B59, 6834 (1999).

17. R.E. Collin, Foundation for Microwave Engineering McGraw-Hill, Inc., New York (1992).
18. I.M. Lifshits, M.Ya. Azbel', and M.I. Kaganov, Electron Theory of Metals, Consultants, Bureau, New York (1973).

19. A. Gordon, I.D. Vagner, and P. Wyder, Adv. Phys. 52, 385 (2003).

20. G. Solt, Solid State Commun. 118, 231 (2001).

21. N. Logoboy, A. Gordon, I.D. Vagner, and W. Joss, Solid State Commun. 134, 497 (2005).

22. W. Joss and N. Logoboy, Ann. Rep. 2005, GHMFL, CNRS (2006).

23. M.I. Tsindlekht, G.I. Leviev, and I. Felner, 6th European Conference on Applied Superconductivity, Sorrento, September 14-18, (2003). Book of Abstracts, p. 28. 